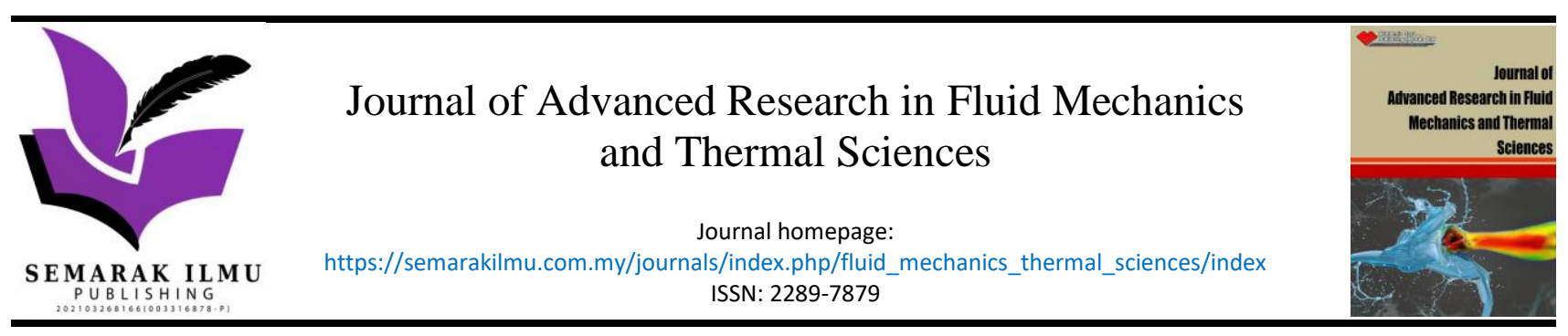

\title{
Flow and Heat Transfer Simulation Analysis of 3D Compact Heat Exchanger
}

\author{
G. Sashwin Nair ${ }^{1,}$, Ahmed N. Oumer ${ }^{1}$, Azizuddin Abd Aziz ${ }^{2}$, Januar Parlaungan Siregar ${ }^{1}$ \\ College of Engineering, Department of Mechanical Engineering, University Malaysia Pahang (UMP), Pahang, Malaysia \\ 2 Faculty of Mechanical and Automotive Engineering Technology, University Malaysia Pahang (UMP), Pahang, Malaysia
}

\section{ARTICLE INFO ABSTRACT}

\section{Article history:}

Received 27 July 2021

Received in revised form 9 September 2021

Accepted 12 September 2021

Available online 7 November 2021
Keywords:

Numerical simulation; Compact heat exchangers; laminar flow; heat transfer; pressure drop
Compact heat exchangers (CHEs) are one of the most commonly used heat exchangers in the industry due to their superior advantages over other types of heat exchangers. Various geometric (fin spacing, tube inclination angle, etc) and process (such as flow velocity, temperature, etc) parameters affect the performance of such compact HEs. This research aims to examine the effects of fin spacing, tube inclination angle, and airflow velocity on heat transfer and pressure drop performance of $\mathrm{CHE}$ in both inline and staggered configurations. A three-dimensional (3D) numerical method with the aid of Ansys FLUENT software was carried out for the laminar flow condition. Based on the obtained results, the highest average heat transfer coefficient was observed at $120^{\circ}$ for both tube arrangements while the lowest average pressure drop penalty is at $30^{\circ}$. Therefore, the recommended inclination angle when high heat transfer is needed is at $120^{\circ}$ while if the pumping power is the major problem, $30^{\circ}$ or $150^{\circ}$ is recommended. based on the London area goodness factor $(j / f), 30^{\circ}$ and $150^{\circ}$ show the highest value for both configurations. The $j / f$ factor decreases with the increase of Reynolds number for both configurations. In addition, $120^{\circ}$ shows the lowest $\mathrm{j} / \mathrm{f}$ which can be due to the high pressure drop.

\section{Introduction}

In this generation, there are a variety of heat exchangers (HE) used in industry and it's selected based on their applications. The most familiar HEs utilized in the industry are shell and tube HE, plate $\mathrm{HE}$, fin-and-tube $\mathrm{HE}$, condensers, boilers and evaporators. For this study, the compact heat exchanger (CHE) was chosen due to its lightweight, small size and cheap. Examples of CHEs that are usually used are plate HE, fin-and-tube HE and spiral HE [1]. In this study, fin-and-tube was selected due to its superior advantage over other types of HE. This HE is regularly used in automobile radiators to improve heat transfer performance due to its greater heat transfer surface area per volume. The flow in this type of HE is normally in the laminar range [2]. There has recently been a lot of research on the effect of geometric and process characteristics on the heat transfer performance of CHE [3-8]. Moreover, the pressure drop has been given attention to the HE performance as higher pressure

\footnotetext{
* Corresponding author.

E-mail address: sashwinnair1997@gmail.com
}

https://doi.org/10.37934/arfmts.88.3.7187 
requires higher cost. Thus, the most challenging part for the researchers is to improve the HE's geometry to ensure enhanced heat transfer across the HE together with low pressure drop.

Firstly, the flat tube's thermal-hydraulic diameter is smaller than a circular tube. Circular tubes have various drawbacks in $\mathrm{HE}$, including increased tube drag and poor heat transfer on the fin behind the tube. This is due to behind the tubes, a low-velocity wake region is formed. These disadvantages can be solved by using oval or flat tubes in HE. Apart from that, it is proven that flat-tube HE gives a higher air-side heat transfer coefficient with a low-pressure drop [9]. The shape of the tube and fin, fin pitch, tube pitch, tube arrangement and tube angle are all geometric parameters whereas air velocity and temperature of the tube are the process parameters that influence the heat transfer performance on CHE. Various experimental and numerical research has been done to investigate the air-side heat and flow performance using different parameters. Zeeshan et al., [10] numerically performed an investigation with inline and staggered tube arrangement to analyze the heat transfer performance of $\mathrm{CHE}$. They found that staggered arrangement shows a higher heat transfer characteristic than inline arrangement as the vortex formation is seen in each row which plays the major role in heat transfer enhancement. Besides that, the most recent research by Unger et al., [11] states that staggered arrangement gives $88.55 \%$ higher heat transfer enhancement than inline arrangement as the flow being deflected improves the thermal mixing. Toolthaisong and Kasayapanand [12] had experimentally investigated the tube inclination angle of CHE on the heat transfer performance. Based on their results, the minimum and maximum heat transfer are at $0^{\circ}$ and $90^{\circ}$. Wang et al., [3] results show that when the tube is rotated at $30^{\circ}$, the heat transfer coefficient is highest. Furthermore, Tang et al., had experimentally and numerically investigated the effect of air inlet angle on air-side heat transfer characteristics. The author's results state that $45^{\circ}$ shows the best heat transfer performance and $90^{\circ}$ gives the lowest pressure drop. Apart from that, based on $j / f$ factor evaluated by the author, $45^{\circ}$ gives the overall best heat transfer performance followed by $60^{\circ}$. Many types of research work on tube arrangement on heat transfer performance were done by many researchers $[7,8,13,14]$. Moreover, numerous studies have been conducted on the effect of various tube angles on CHE heat transfer performance $[3,4,12,15]$. The study related to the tube arrangement and tube angle has been done separately. However, the combination of both studies is something new and has not been fully analyzed in comparison with plain fin for an enhanced heat transfer performance.

The objective of this research is to see how tube inclination angle affects heat transfer performance in $\mathrm{CHE}$ with inline and staggered tube arrangements. With six tube inclination degrees $\left(0^{\circ}, 30^{\circ}, 60^{\circ}, 90^{\circ}, 120^{\circ}, 150^{\circ}\right)$, a numerical method was developed in ANSYS FLUENT software. This study would help engineers to decide the angle of inclination and design the CHE for an improved heat transfer performance with low pressure drop. Moreover, engineers that are designing a high energy efficiency CHE might also be helpful from this study.

\section{Methodology}

\subsection{Method and Materials}

The schematic diagram of fin and tube cross-sections used in this study is shown in Figure 1. Tube arrangements considered in this study are in-line and staggered configurations. Air flowed across the surface of the fin and tubes and constant heat flux was contributed from the surfaces of the inner tube. The HE's model consists of an overall length of the flat tube of $220 \mathrm{~mm}$ and $15.1 \mathrm{~mm}$ tube hydraulic diameter. Square-shaped fins were used with the dimension of $110 \mathrm{~mm} \times 110 \mathrm{~mm}$ and 0.6 $\mathrm{mm}$ thickness while Table 1 shows the other geometric parameters of the HE whereas Table 2 shows 
the properties of air. In this study, the geometric parameters of the HE are considered as previous work done by Adam et al., [15].

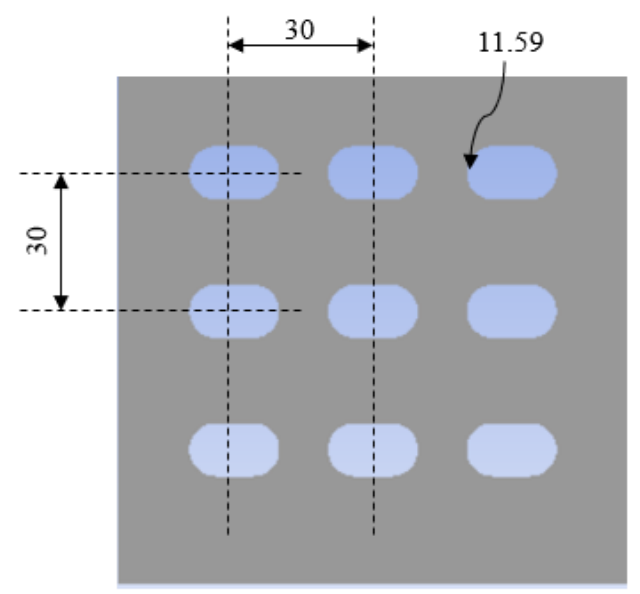

(a)

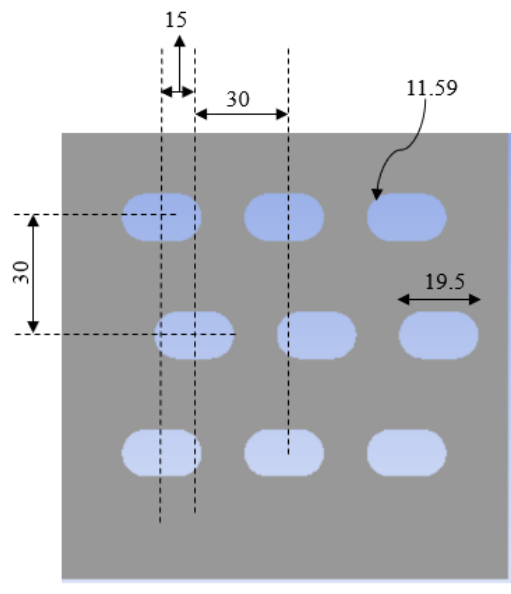

(b)

Fig. 1. Schematic diagrams of the cross-section of HE with (a) in line and (b) staggered arrangement. (Values in $\mathrm{mm}$ )

Table 1

Geometric Parameters of HE

\begin{tabular}{lll}
\hline Name & Value $(\mathrm{mm})$ & Symbol \\
\hline Transverse pitch & 30 & $P_{t}$ \\
Longitudinal pitch & 30 & $P_{l}$ \\
The outside diameter of & 11.59 & $D_{o}$ \\
the tube & & \\
The hydraulic diameter of & 15.1 & $D_{h}$ \\
the tube & 0.6 & $t$ \\
Thickness of fin & 20 & $F_{s}$ \\
Spacing of fin & - & $A l$ \\
Material of tube and fin & &
\end{tabular}

Table 2

Properties of Air

\begin{tabular}{lll}
\hline Name & Value & Symbol \\
\hline Density & $1.225 \mathrm{~kg} / \mathrm{m}^{3}$ & $\rho$ \\
Specific heat & $1006.43 \mathrm{~J} / \mathrm{kg} . \mathrm{K}$ & $c_{p}$ \\
Thermal conductivity & $0.0242 \mathrm{~W} / \mathrm{m} . \mathrm{K}$ & $k$ \\
Viscosity & $1.7894 \times 10^{-5} \mathrm{~kg} / \mathrm{m} . \mathrm{s}$ & $\mu$ \\
\hline
\end{tabular}

\subsection{Computational Domain}

The computational domain of 3 rows by 3 columns for both tube configurations is shown in Figure 2. At the upstream, the computational domain was extended 2 times the tube outer diameter $\left(D_{0}\right)$ to form a fully developed constant velocity flow. Besides, the domain was extended 5 times the tube outer diameter to avoid airflow recirculation and to make sure the boundary layer formation at the outlet is fully developed [8]. A 2D view of the inlet and outlet extension with boundary conditions is shown in Figure 3. Symmetry conditions were used in right, left, top and bottom of the test section. 


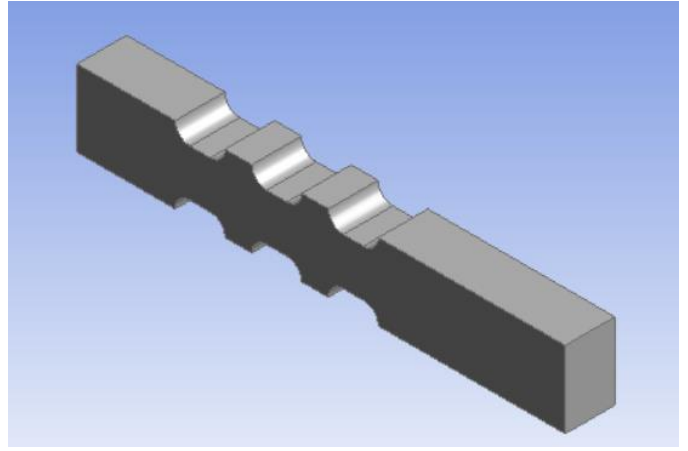

(a)

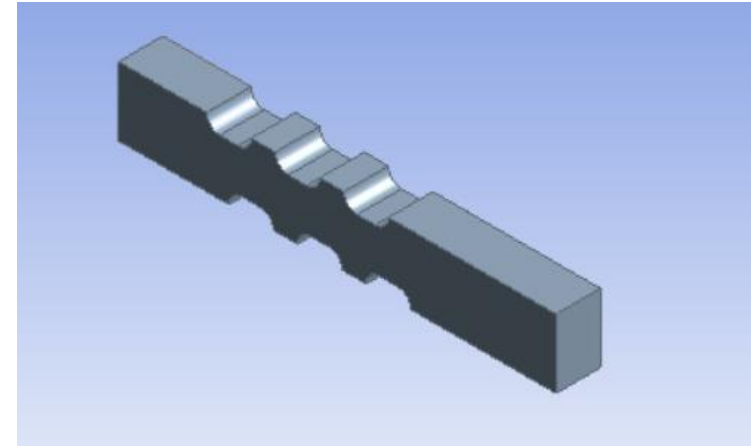

(b)

Fig. 2. Computaional region of a) in-line and b) staggered arrangement

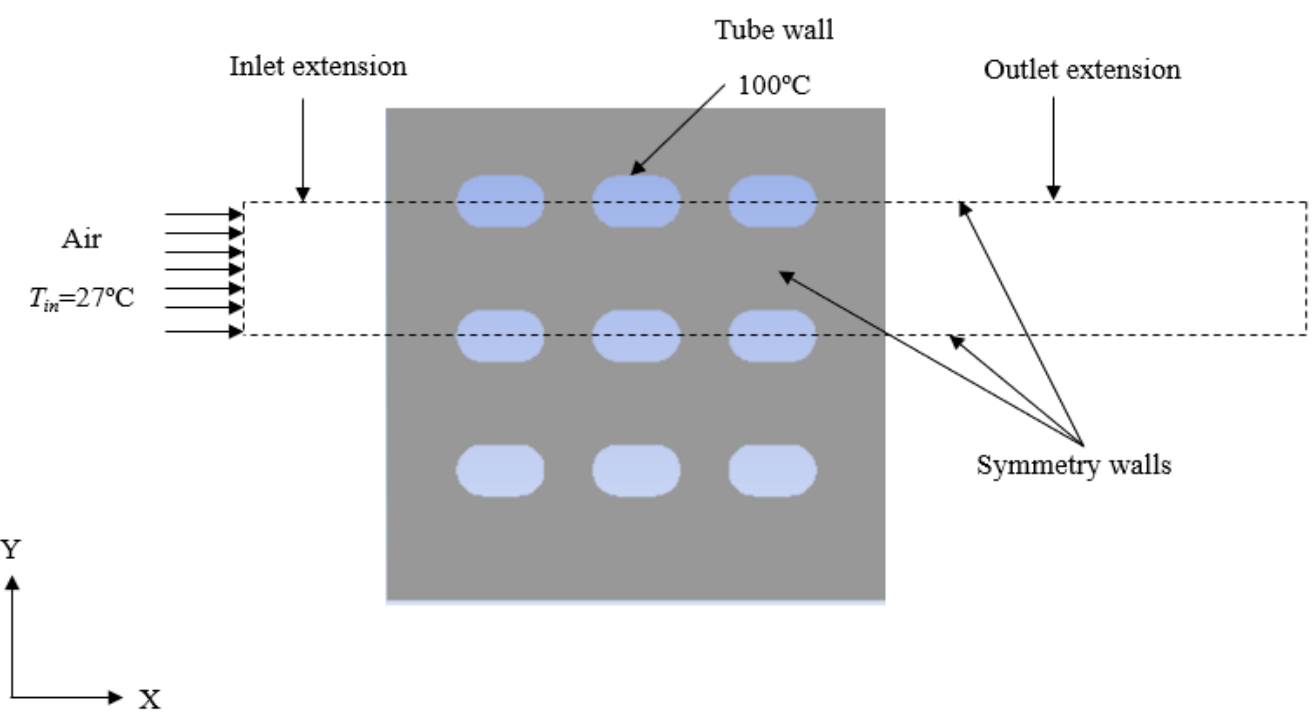

(a)

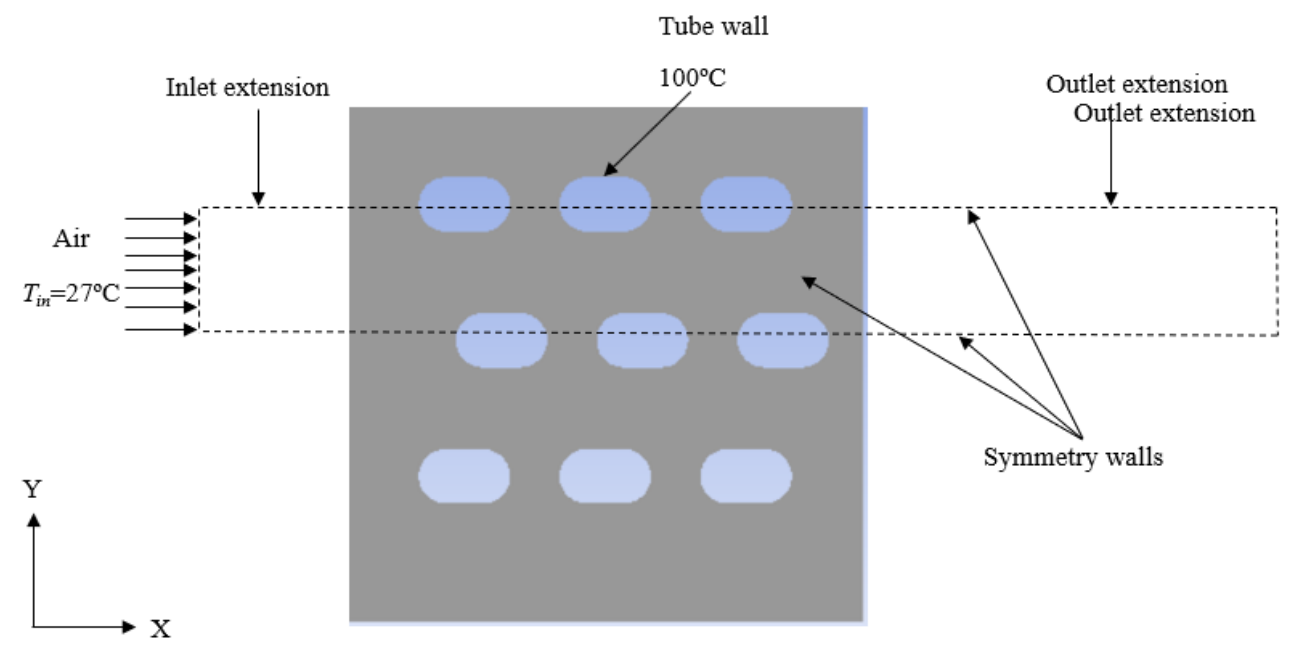

(b)

Fig. 3. 2D view of computational domain with boundary conditions for (a) in-line and (b) staggered arrangement 


\subsection{Mathematical Equation}

\subsubsection{Governing equation}

The numerical model assumes that the stream region is incompressible, turbulent, nonisothermal, and consistent. In addition, physical parameters are assumed to be constant, thermal radiation and heat dissipation are ignored, and the fin surfaces are assumed to be smooth. These assumptions are used to define the continuity, momentum, and energy governing equations, as well as the transport equation of turbulent kinetic energy and its dissipation rate. The following are the equations for continuity, momentum, and energy [16].

$\nabla .(V)=0$

$\rho \nabla \cdot(\boldsymbol{V} \boldsymbol{V})=-\nabla P+\nabla \cdot \tau$

$\rho C p D \cdot(T V)=k V \cdot T$

\subsubsection{Parameters definitions}

Heat transfer and pressure drop characteristics for compact HE are determined by the airflow and design of the HE. In the equation below, the heat transfer coefficient between the finned flat tube and air is shown below [14]

$h=\frac{\dot{Q}}{A\left(T_{s}-T_{b}\right)}$

$T_{b}=\frac{T_{\text {in }}+T_{\text {out }}}{2}$

where $\dot{Q}$ is the heat transfer rate, $A$ is the total surface area including fins and tubes, $T_{S}$ is the tube wall surface temperature and $T_{b}$ is bulk fluid temperature.

The pressure drop is the difference between the outlet and inlet pressure of the flow, written as [7]:

$\Delta P=P_{\text {in }}-P_{\text {out }}$

Furthermore, the friction factor is also a vital aspect where it determines the non-dimensional pressure drop of the flow as shown below

$f=\frac{2 D_{h} \Delta P}{L \rho V_{\max }^{2}}$

where $D_{h}$ is the hydraulic diameter, $L$ is the airflow length, $V$ is the airflow velocity and $\rho$ is the density of air.

The Colburn factor is also considered in this study since heat transfer is involved in the investigation. It is a correlation of heat, momentum, and mass transfer for convective heat transfer [17].

$j=\frac{N u}{\operatorname{RePr}^{1 / 3}}$ 
where $N u$ is Nusselt number, $R e$ is Reynolds number and $P r$ is Prandtl number.

Nusselt number is the ratio across the boundary of natural convective heat transfer [13].

$N u=\frac{h D_{h}}{k}$

where $k$ is the thermal conductivity.

Prandtl number is a parameter that displays the ratio of viscous diffusivity to thermal diffusivity, given as [3]

$\operatorname{Pr}=\frac{c_{p} \mu}{k}$

where $c_{p}$ is the specific heat at constant pressure.

The overall thermal-hydraulic performance was calculated to compare the airside performance. Therefore, the performance evaluation criteria of CHE was defined as [8]

$P E C=J F=\frac{j}{f}$

\subsection{Meshing}

ANSYS Fluent 19.2 is used to perform the numerical analysis of the governing equations. In this study, the symmetry boundary condition was used to reduce the number of grids, resulting in a reduction in calculation time. $373 \mathrm{~K}$ is maintained on the tube surfaces, while $300 \mathrm{~K}$ is maintained on the inlet air velocity. Inlet velocity in the range of 1.8 to $3.8 \mathrm{~m} / \mathrm{s}$ was assigned to the inlet section of Figure 3. Figure 4 depicts the grid generation process used in this research. The hexahedral element was chosen for this study because it is uniform and smooth, ensuring numerical prediction and low processing cost which was also chosen by Nguyen Minh Phu and Pham Ba Thao [18].

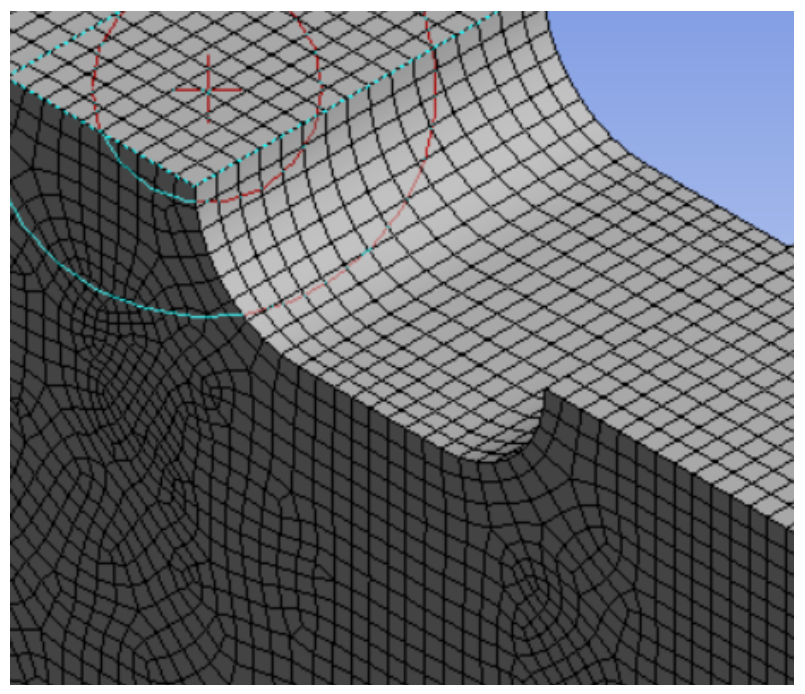

Fig. 4. Grid generation 
The boundary conditions used in the computations are shown as follows

i. A uniform velocity and temperature $(300 \mathrm{~K})$ are fixed at the inlet boundary.

ii. All tube walls have no-slip conditions. The test section's lateral surfaces are given symmetry conditions.

iii. The temperature of the tube walls is set to $373 \mathrm{~K}$.

iv. The computational domain is expanded downstream for a fully developed outlet boundary state.

v. Other surfaces are assigned symmetrical boundary conditions.

\subsubsection{Mesh metrics quality}

Mesh Metrics are the most practical quality to determine the accurate shape and size of the elements. Before proceeding to the mesh independency test, the mesh metrics quality needs to be in the appropriate range. In this study, orthogonal, skewness and aspect ratio were taken into consideration. The range for good mesh metrics are as follows

i. Orthogonal $=\min >0.25$

ii. $\quad$ Skewness $=\max <0.8$

iii. Aspect ratio $=\max <40$

Based on table 3, the value for each mesh metric is in the allowable range. Thus, the quality of the mesh is acceptable.

\section{Table 3}

Mesh metrics quality

\begin{tabular}{lllll}
\hline Mesh & Number of grids & \multicolumn{3}{l}{ Mesh Metrics Quality } \\
\cline { 3 - 5 } & & Orthogonal & Skewness & Aspect Ratio \\
1 & 55605 & Min $=0.47692$ & Max $=0.62748$ & Max $=11.321$ \\
3 & 60660 & Min $=0.47692$ & Max $=0.62748$ & Max $=11.182$ \\
4 & 65715 & Min $=0.47692$ & Max $=0.62748$ & Max $=10.24$ \\
5 & 93092 & Min $=0.54988$ & Max $=0.57556$ & Max $=8.0932$ \\
\hline
\end{tabular}

\subsubsection{Mesh independency test}

The mesh independency test assists in determining the model's acceptable amount of tolerance based on meshing size [19]. In this study, the mesh independency test was carried out with 5 different grid sizes which are 55605, 60660, 65715, 93092 and 109000. In this test, in-line arrangement with $1.8 \mathrm{~m} / \mathrm{s}$ inlet velocity was kept constant. The mesh independency test for the five different grid sizes is shown in Figure 4. The difference in velocity between mesh 93092 and 109000 is shown to be 7.88 $\%$, which is less than $10 \%$. The outcome is acceptable as it saves computational time and space without compromising the simulation results. Thus, the finalize grid size used in this study is 93092. 


\section{Mesh Independency Results}

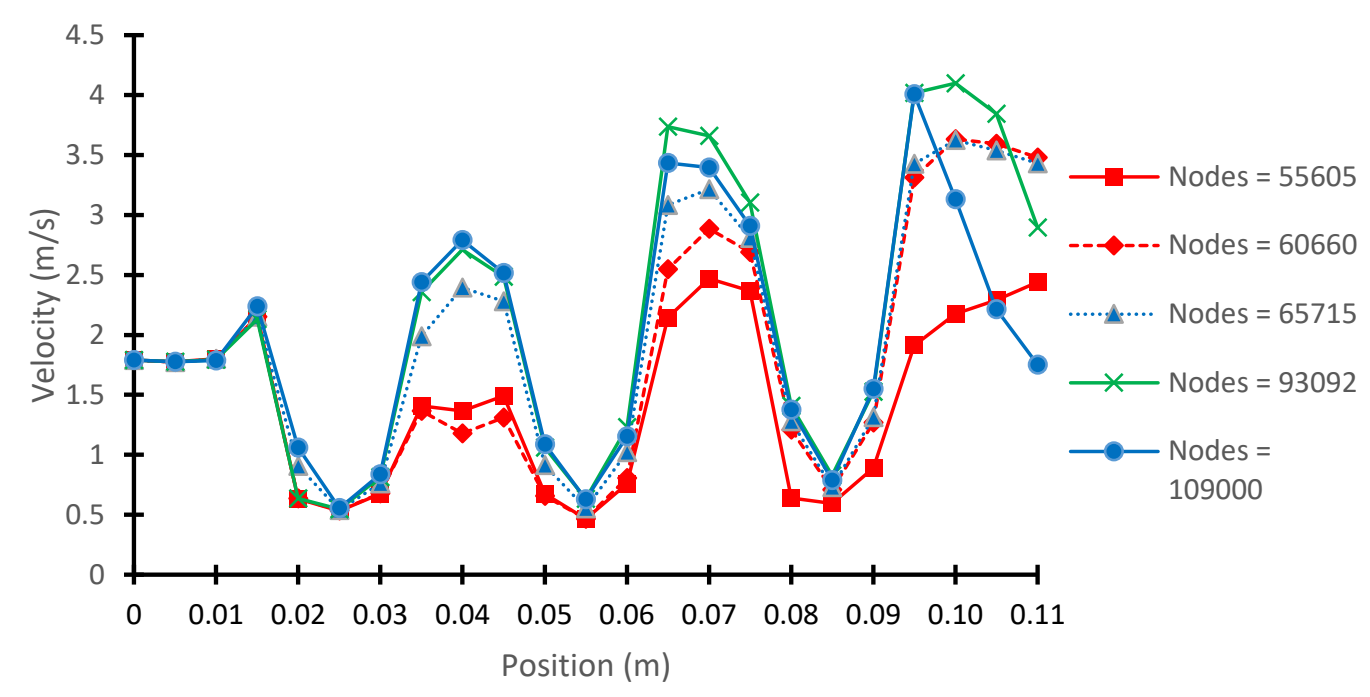

Fig. 5. Mesh independency test

\subsection{Model Validation}

The validity of the numerical model was examined as the outlet temperature of the model was compared with the results presented by Adam [20]. Figure 6 shows the variation of outlet temperature with inlet air velocity. The maximal deviation between the outlet temperature of the present study and experimental results was $9.8 \%$. This clearly shows that the outlet temperature value of the present study lies within the allowable range of error as several previous research error shows more than $9.8 \%$ and they assume the model is valid. $[7,8,15]$. Therefore, good acceptance between the present study and previous research study shows that the numerical model is valid.

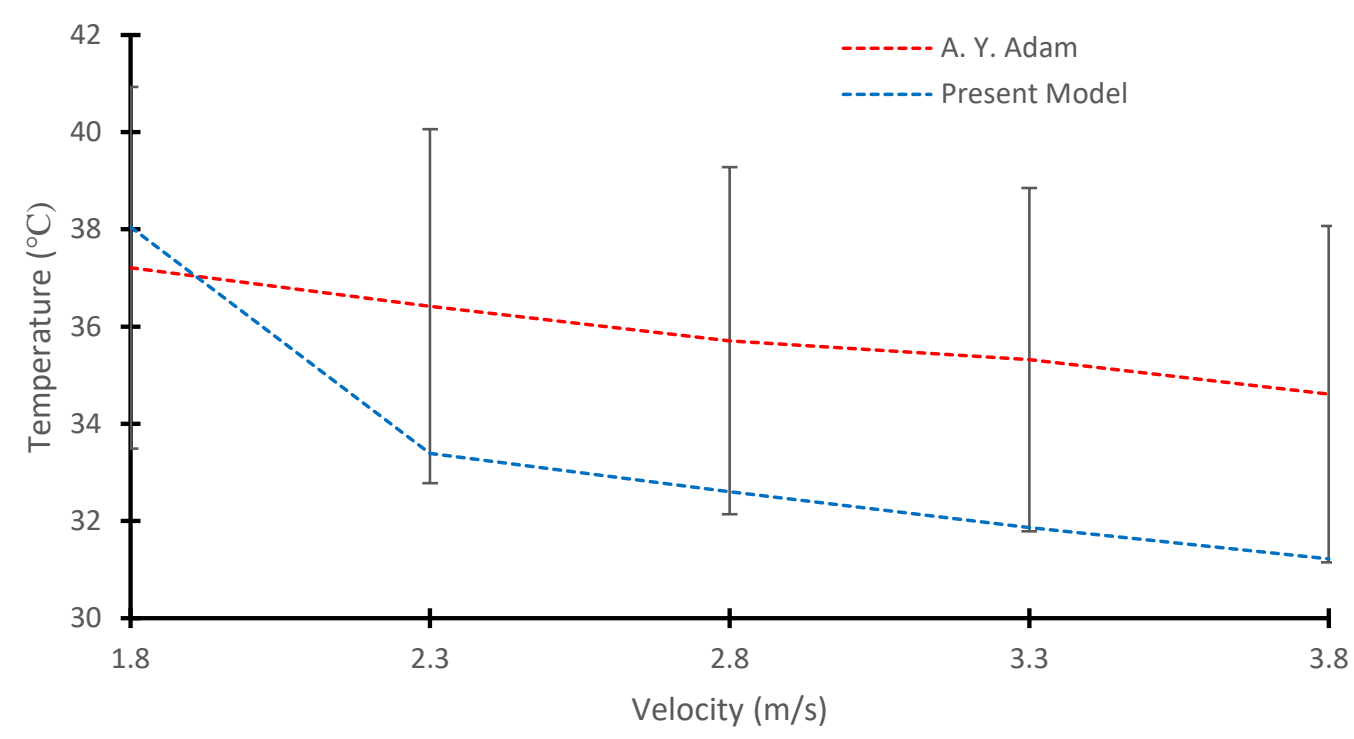

Fig. 6. Model validation 


\section{Results}

\subsection{Flow and Temperature Visualization}

\subsubsection{Flow visualization}

Figure 7 and 8 show the influence of tube inclination angle on the flow distribution for both inline and staggered tube configurations at a velocity of $v=1.8 \mathrm{~m} / \mathrm{s}$. Recirculation occurs after each tube in both inline and staggered tube arrangements, according to the results. Due to the blockage of the tube, when air flows across the fin, flow separation occurs. Except for tube inclination angles of $30^{\circ}$, $60^{\circ}$, and $90^{\circ}$, when the flow separates at the back of the tube, it bounces back at the frontal area of the next tube, resulting in a bigger region. This study mainly to figure out the air-side thermal performance of the CHE rather than the fin-side. The contours displayed in Figures 7 and 8 are not the fin but the spacing between the two fins. This can be the reason why the back of the successive tube doesn't show a bigger region from the flow separation. Furthermore, the flow velocity is higher at the tube wall region which makes that region effective for the heat transfer process [21].

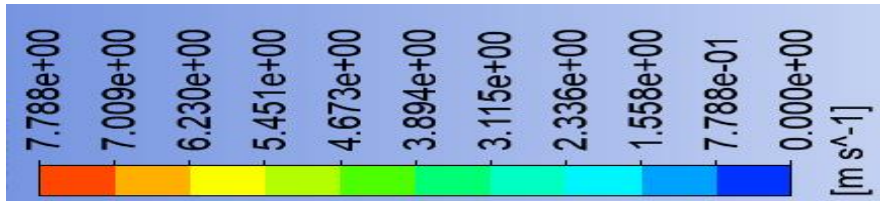

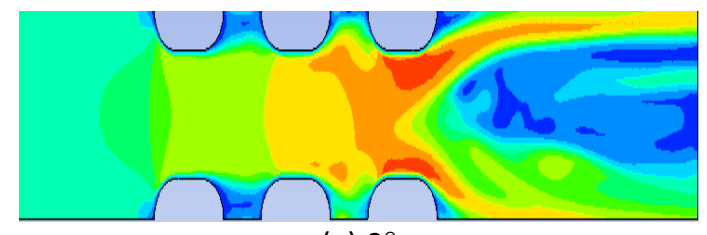

(a) $0^{\circ}$

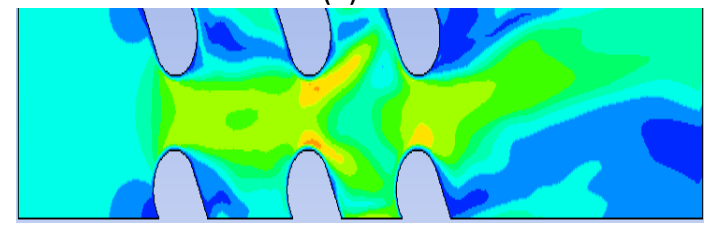

(c) $60^{\circ}$

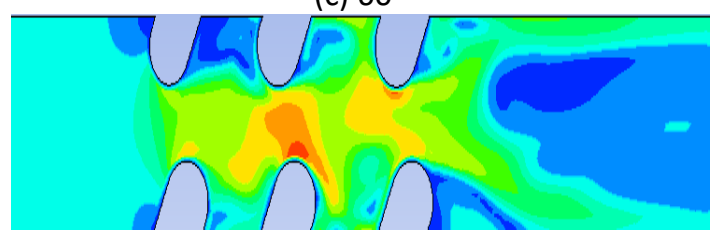

(e) $120^{\circ}$

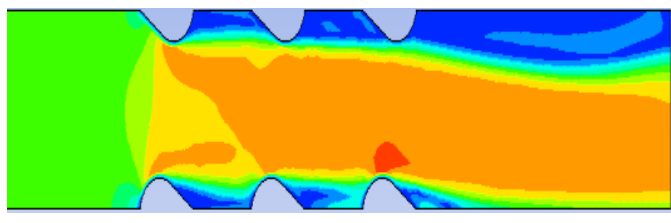

(b) $30^{\circ}$

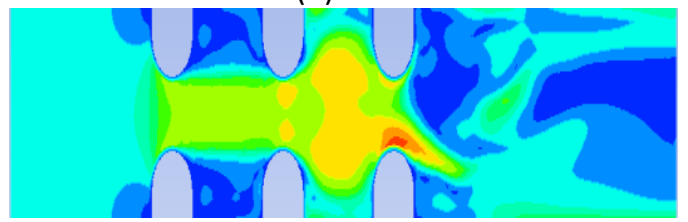

(d) $90^{\circ}$

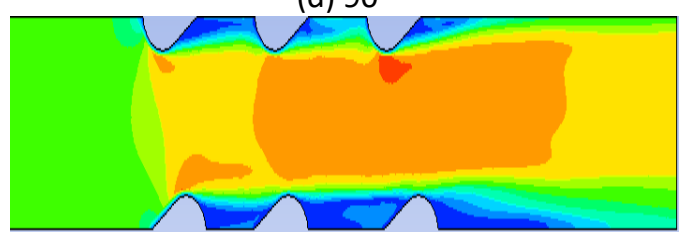

(f) $150^{\circ}$

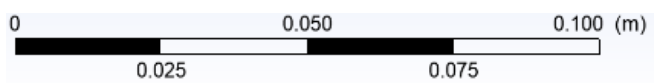

Fig. 7. Velocity distribution for inline arrangement at $\mathrm{v}=1.8 \mathrm{~m} / \mathrm{s}$ 


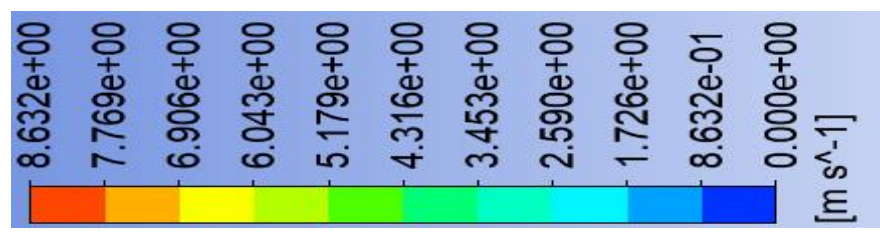

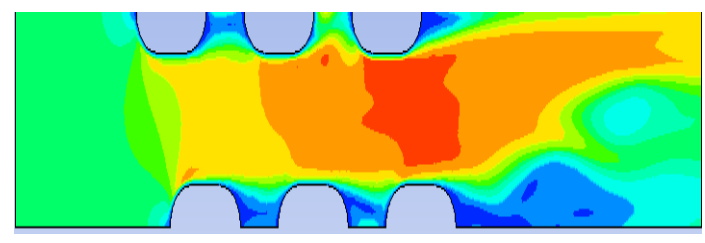

(a) $0^{\circ}$

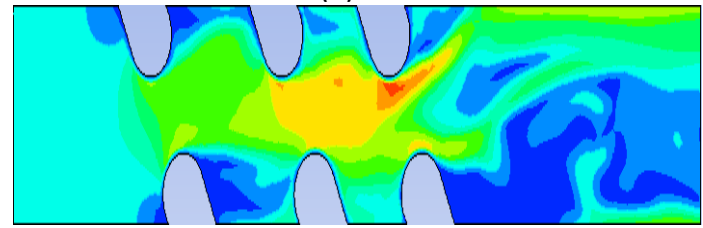

(c) $60^{\circ}$

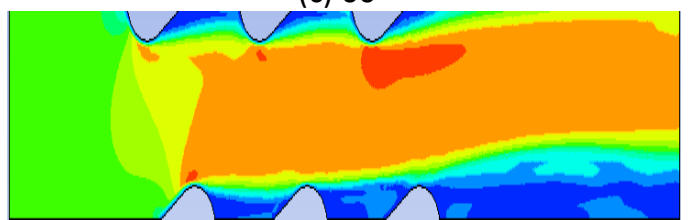

(e) $120^{\circ}$

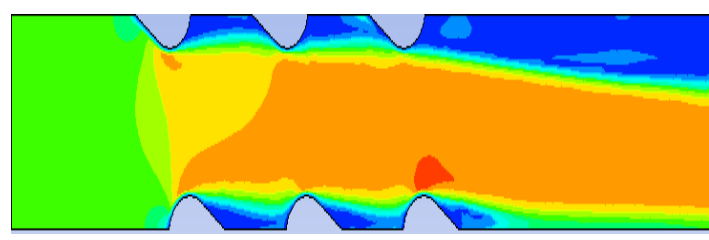

(b) $30^{\circ}$

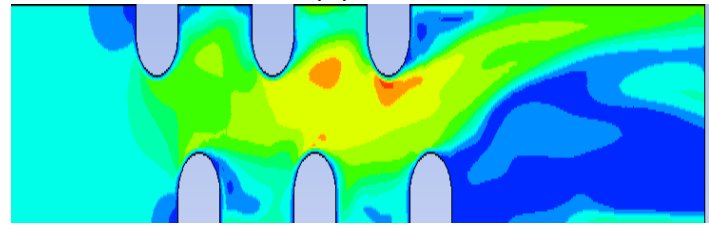

(d) $90^{\circ}$

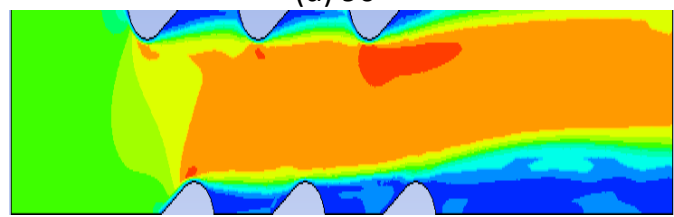

(f) $150^{\circ}$

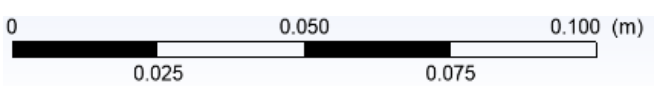

Fig. 8. Velocity distribution for staggered arrangement at $v=1.8 \mathrm{~m} / \mathrm{s}$

\subsubsection{Temperature visualization}

Figure 9 and 10 show the influence of tube inclination angle on the temperature distribution for both inline and staggered tube arrangements at an airflow velocity of $v=1.8 \mathrm{~m} / \mathrm{s}$. Based on the temperature contours below, before the flow reached the tube, the entire inlet region is at the same temperature which is at $300 \mathrm{~K}$. Apart from that, it is expected as the temperature is highest at the tube wall region. Based on the figures, the outlet temperature of all the tube inclination angle is different. Expanding the thermal boundary layer along the tube wall region is one of the most important factors in improving heat transfer. For inline arrangement, except for $90^{\circ}$, the boundary layer thickness at the $3^{\text {rd }}$ row of the tube is larger than the $1^{\text {st }}$ row of the tube because of the larger air velocity around the $1^{\text {st }}$ row tube before it travels to the $3^{\text {rd }}$ row of the tube [21]. Moreover, at $90^{\circ}$, more incoming air is blocked as the $3^{\text {rd }}$ row of the tube shows a smaller heat distribution compared to $0^{\circ}$. 


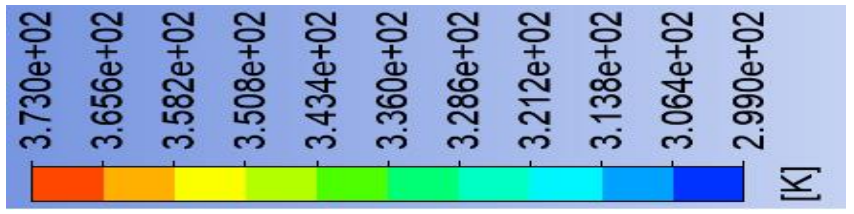

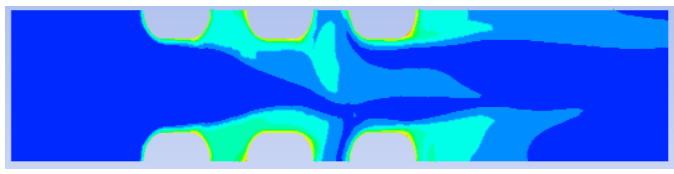

(a) $0^{\circ}$

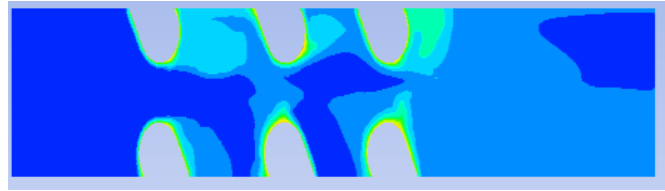

(c) $60^{\circ}$

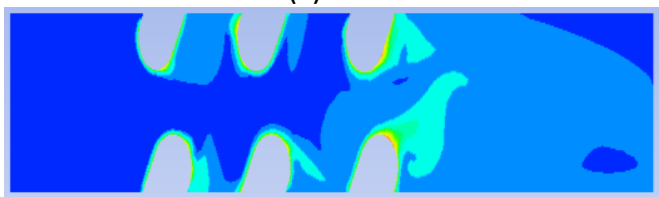

(e) $120^{\circ}$

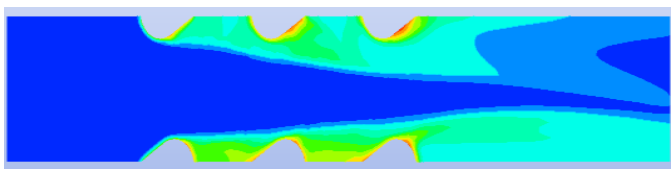

(b) $30^{\circ}$

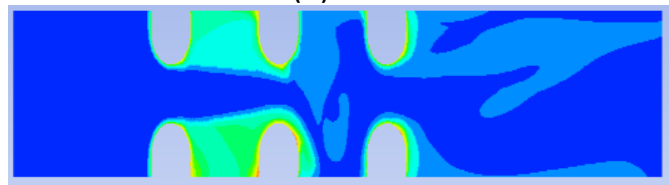

(d) $90^{\circ}$

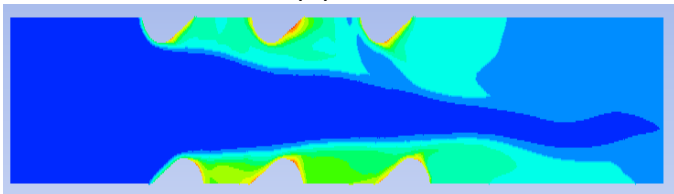

(f) $150^{\circ}$

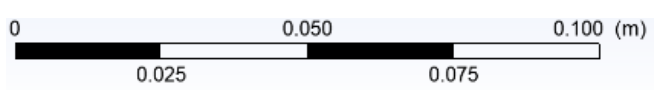

Fig. 9. Temperature distribution for inline arrangement at $v=1.8 \mathrm{~m} / \mathrm{s}$

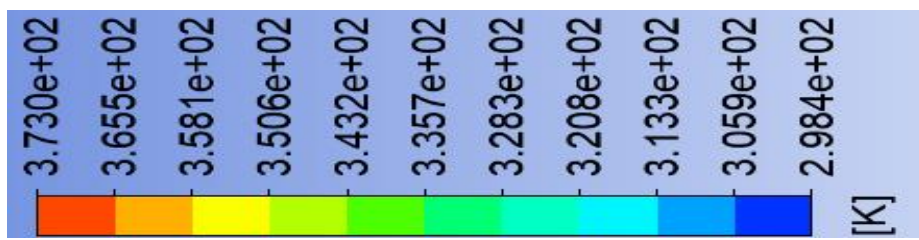

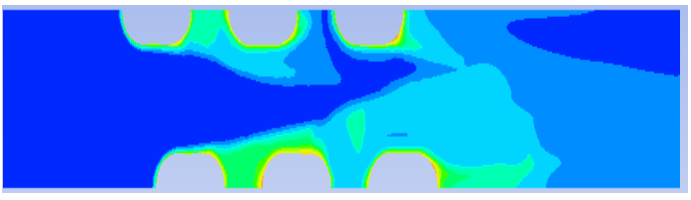

(a) $0^{\circ}$

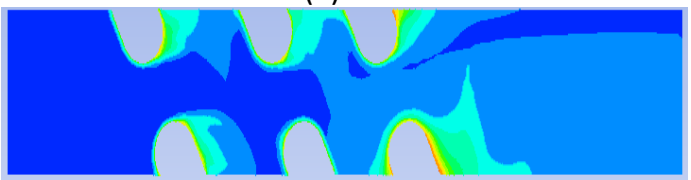

(c) $60^{\circ}$

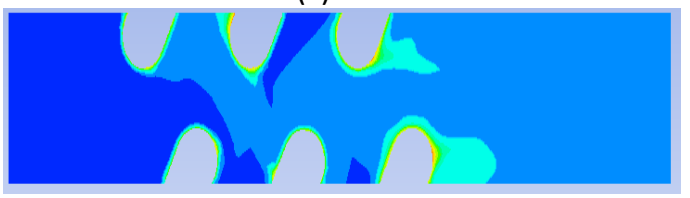

(e) $120^{\circ}$

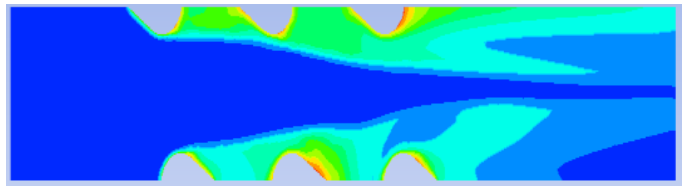

(b) $30^{\circ}$

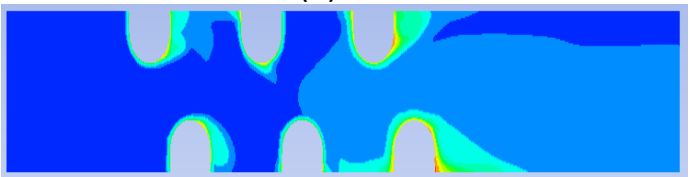

(d) $90^{\circ}$

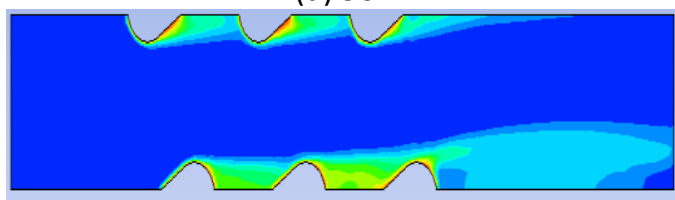

(f) $150^{\circ}$

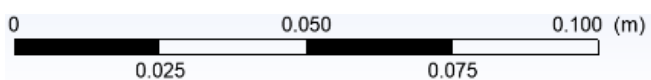

Fig. 10. Temperature distribution for staggered arrangement at $\mathrm{v}=1.8 \mathrm{~m} / \mathrm{s}$ 


\subsection{Flow and Heat Transfer Performance}

\subsubsection{Variation of $h$ with inlet $v$ at $0^{\circ}$ inclination angle for inline and staggered arrangement}

Figure 11 shows the variance of heat transfer coefficient with inlet velocity for both arrangements at $0^{\circ}$ tube inclination angle. As illustrated in the results, the heat transfer coefficient for inline configuration increases with the inlet airflow velocity, whereas the heat transfer coefficient for staggered arrangement increases until $3.3 \mathrm{~m} / \mathrm{s}$ and then slightly declines until $3.8 \mathrm{~m} / \mathrm{s}$. Higher velocity disturbs the thermal boundary layer more because the vortices produced by the higher velocity are stronger. In comparison with inline and staggered, inline arrangement shows a higher average heat transfer coefficient than staggered arrangement. This could be because the tube around the staggered configuration has an uneven flow [15].

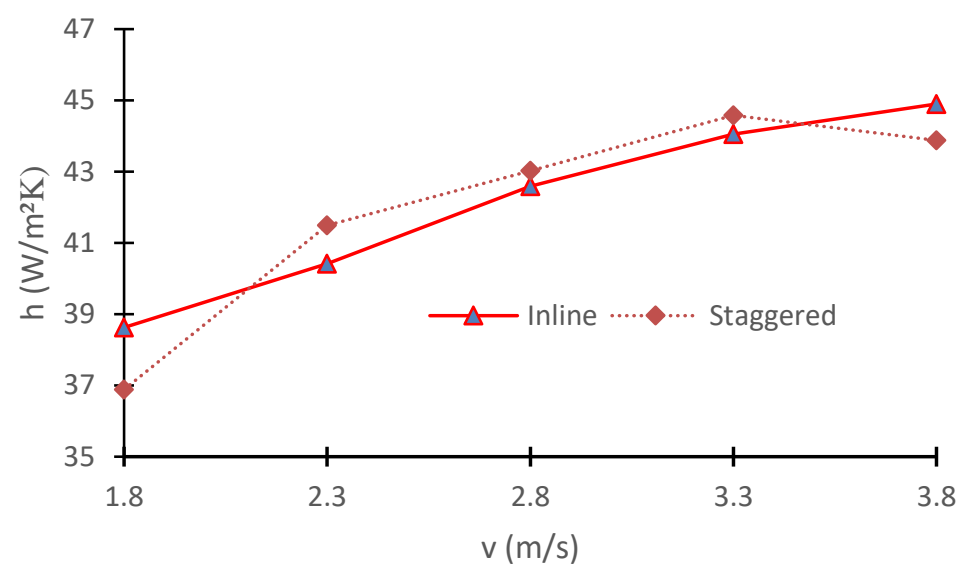

Fig. 11. Heat transfer coefficient against inlet velocity $v$ at $0^{\circ}$

\subsubsection{Effect of inlet velocity on $\Delta P$}

Figure 12 depicts the difference of pressure drop with inlet velocity for both inline and staggered arrangements at a $0^{\circ}$ tube inclination angle. Based on Figure 12, in both inline and staggered arrangement, when the inlet velocity increases, the pressure drop also increases. Hoffmann et al., stated that the static pressure changes from one tube row to another. Higher velocities passing through the fin create a larger segment of static pressure [22]. It is observed that the staggered arrangement gives a lower average pressure drop than the inline arrangement. This phenomenon might be because, during the recirculation zone, two different types of forces are formed which are pressure and drag forces. This might be the reason for the pressure loss in the recirculation region. 


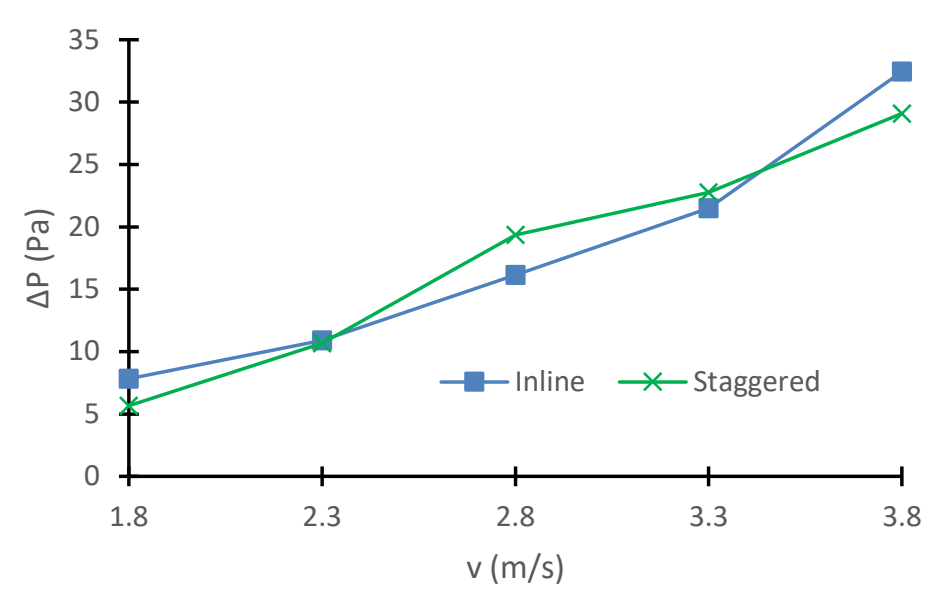

Fig. 12. Pressure drop against inlet velocity $v$ at $0^{\circ}$

\subsubsection{Effect of tube inclination angle on $h$}

Variations of heat transfer coefficient for inline and staggered arrangements with different tube inclinations at a constant velocity of $v=2.8 \mathrm{~m} / \mathrm{s}$ are provided in this section. As shown in Figure 13, the heat transfer coefficients for different tube inclination angles have different values. For both inline and staggered arrangement, when the inclination is at $120^{\circ}$, it gives the highest heat transfer coefficient This could be related to a delay in flow separation, resulting in a steady and stable reduction in the wake zone behind the tube. [8]. As predicted, the heat transfer coefficient value for $30^{\circ}$ and $60^{\circ}$ were almost the same with $150^{\circ}$ and $120^{\circ}$ respectively which is the same as the results claimed by Adam et al. [15]. Apart from that, the average heat transfer coefficient for inline arrangement is higher than the staggered arrangement. This phenomenon can be due to the blockage of air when it passes through the computational domain in a staggered arrangement. Therefore, less heat transferred air passes through the outlet region.

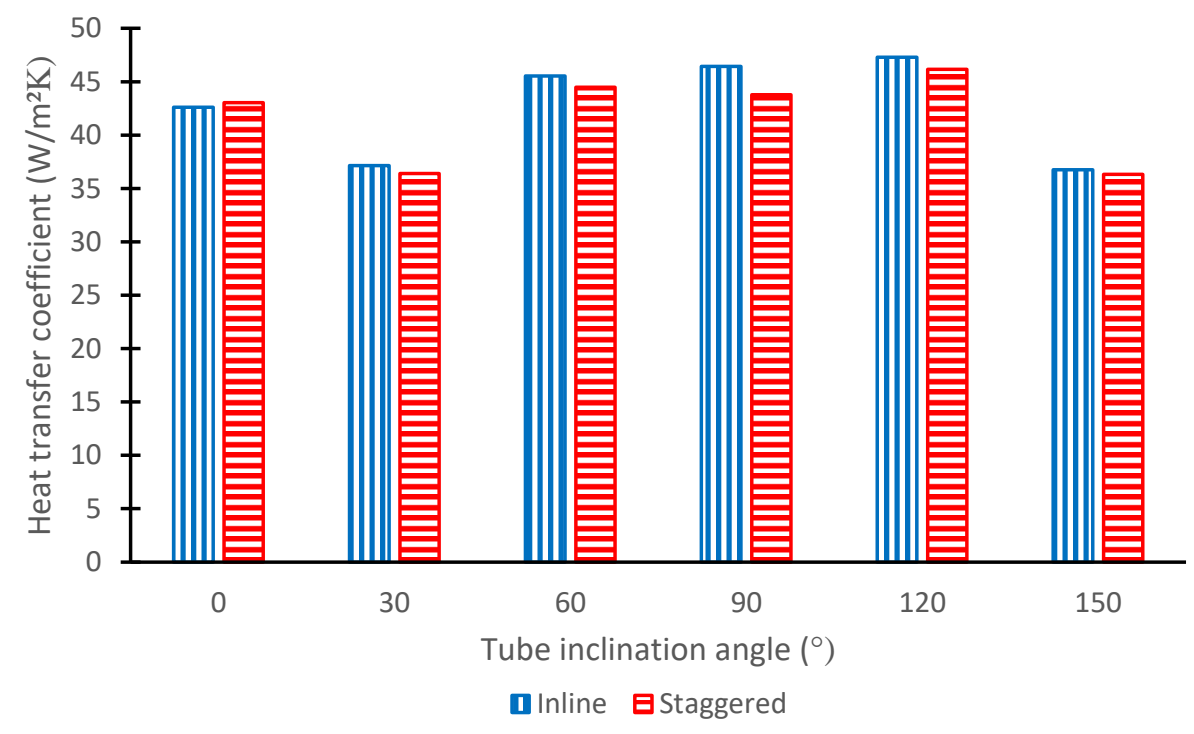

Fig. 13. Heat transfer coefficient against tube inclination angle at $v=2.8 \mathrm{~m} / \mathrm{s}$ 


\subsubsection{Effect of tube inclination angle on $\Delta P$}

This section describes the variation of pressure drop with $\mathrm{v}=2.8 \mathrm{~m} / \mathrm{s}$ at various tube inclinations for inline and staggered arrangements. Based on Figure 14, it is obvious that the results have a different value of pressure drop for different tube inclination angles. For inline arrangement, $120^{\circ}$ and $30^{\circ}$ gives the highest and lowest pressure drop respectively while for staggered arrangement, $90^{\circ}$ and $150^{\circ}$ gives the highest and lowest pressure drop. Comparing both inline and staggered configurations, Figure 14 shows that the average pressure drop is lower for staggered arrangements. The high value of pressure drop obtained might be because the tube's surface area intersects the airflow is large which gives extra drag and causing blockage of airflow by the tube [15]. In conclusion, the heat transfer can be enhanced by tilting the tubes at a certain angle together with a high pressure drop.

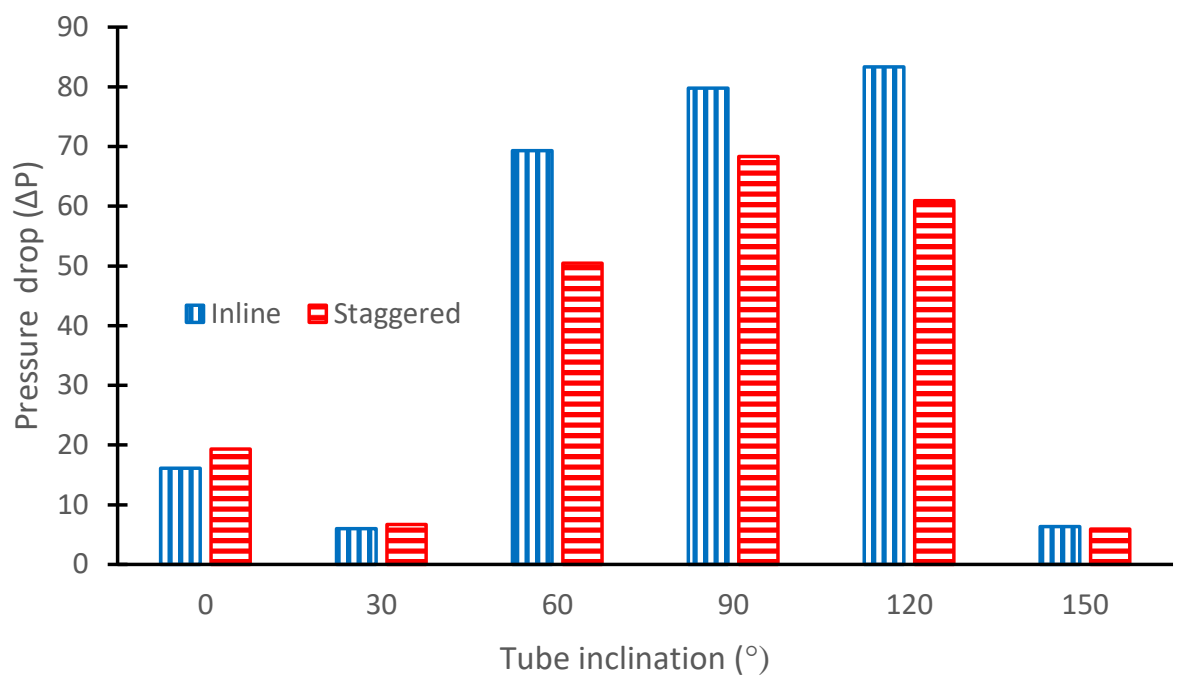

Fig. 14. Pressure drop against tube inclination angle at $v=2.8 \mathrm{~m} / \mathrm{s}$

\subsubsection{Effect of tube inclination angle on London area goodness factor (j/f)}

It is important to evaluate the overall thermal-hydraulic performance of the CHE with various tube inclination angles. The overall thermal-hydraulic performance can be evaluated by using the London area goodness factor (JF factor) which is the ratio of Colburn factor and friction factor. Based on the JF factor, the higher the value of $j / f$, the higher the rate of heat transfer with lower pressure drop. This section explains the variation of $j / f$ with various tube inclination angles for both inline and staggered arrangements. Figure 15 shows the thermal-hydraulic performance of CHE with various tube inclination angle for inline and staggered arrangement. The $j / f$ factor decreases with the increase of Reynolds number for both configurations. In comparison with all the tube inclination angles, $\mathrm{j} / \mathrm{f}$ for $30^{\circ}$ and $150^{\circ}$ are the highest for both configurations. It is expected that the $\mathrm{j} / \mathrm{f}$ for 30 and 150 and 60 and 120 are almost the same to each other. Furthermore, $120^{\circ}$ shows the lowest $\mathrm{j} / \mathrm{f}$ which can be due to the high pressure drop even though it shows the highest heat transfer coefficient. 


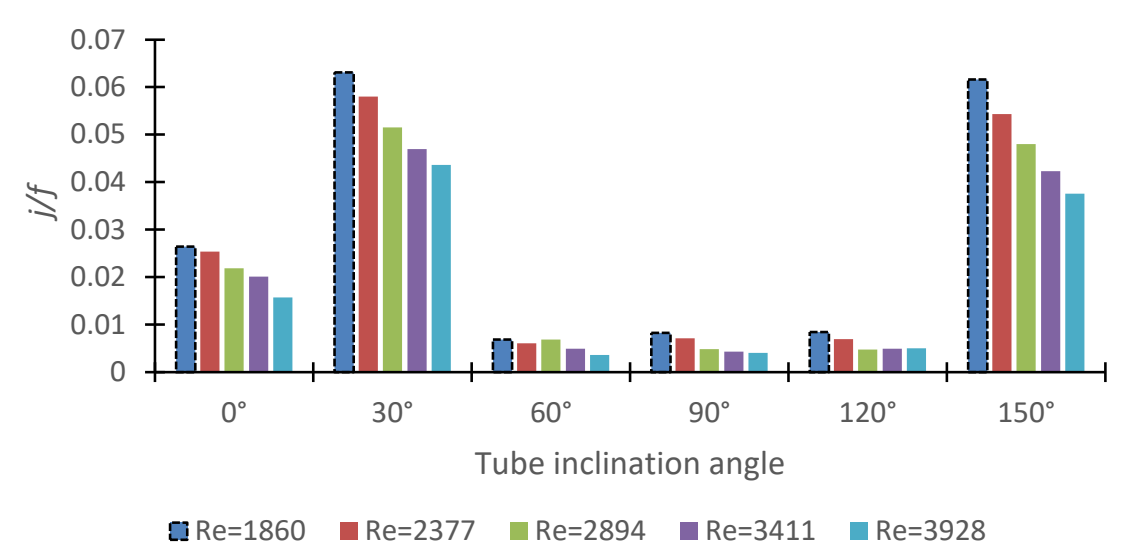

(a)

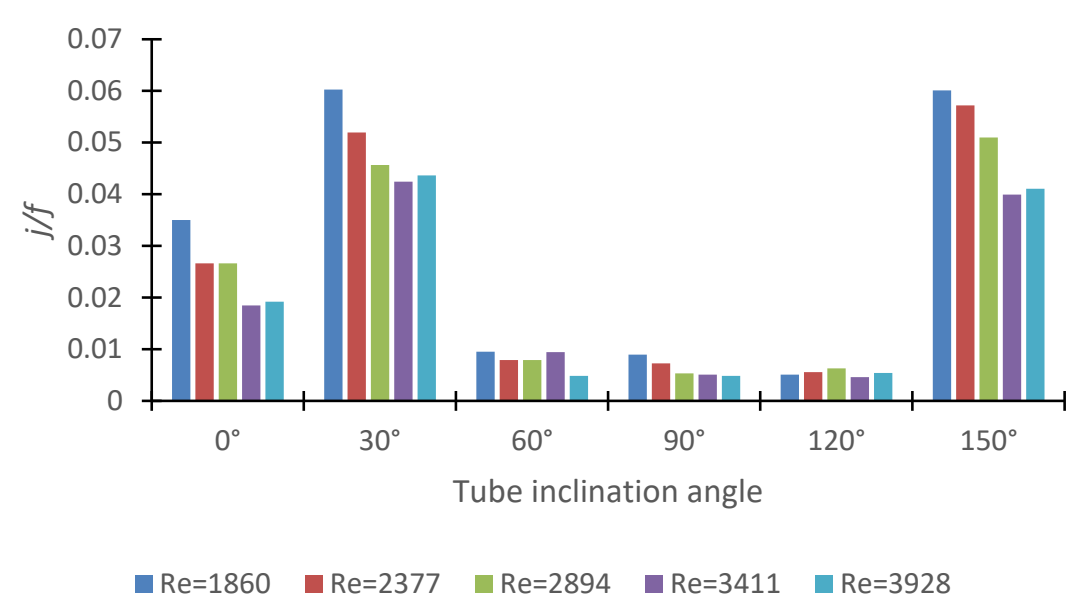

(b)

Fig. 15. Variations in London area goodness factor $(j / f)$ with different tube inclination angles for (a) inline and (b) staggered arrangement

\section{Conclusions}

The heat transfer efficiency and pressure drop of inline and staggered tube arrangement $\mathrm{CHE}$ with various tube inclination angles were investigated using $3 \mathrm{D}$ numerical simulations in this research. The maximum and minimum heat transfer coefficients were found to be $120^{\circ}$ and $30^{\circ}$, respectively, according to the numerical results. Additionally, for both configurations, the $120^{\circ}$ tube inclination angle provides the best heat transfer and pressure drop. Thus, $120^{\circ}$ is recommended when researchers or engineers require a high heat transfer in their work. Apart from that, if the pumping power is the main issue, $30^{\circ}$ or $150^{\circ}$ tube inclination is recommended. The tube inclination angle, according to this research, has a key effect in heat transfer augmentation, such as flow separation delay and reduction in the wake region behind the tube. As a final result, an enhanced heat transfer can occur. In a comparison of inline and staggered arrangement, both give almost the same heat transfer performances. Thus, for the pressure drop penalty, the staggered arrangement shows a lower pressure drop than the inline arrangement. Lastly, based on the London area goodness factor $(j / f), 30^{\circ}$ and $150^{\circ}$ show the highest value for both configurations. The $j / f$ factor decreases with the increase of Reynolds number for both configurations. In addition, $120^{\circ}$ shows the lowest $\mathrm{j} / \mathrm{f}$ which can be due to the high pressure drop even though it shows the highest heat transfer coefficient. 


\section{Acknowledgment}

The authors are grateful to the University of Malaysia Pahang for providing the requisite support through the UMP Fundamental Research Grant scheme under project no. RDU190319.

\section{References}

[1] Zohuri, Bahman. "Heat exchanger types and classifications." In Compact Heat Exchangers, pp. 19-56. Springer, Cham, 2017. https://doi.org/10.1007/978-3-319-29835-1 2

[2] Cengel, Yunus A., and Afshin J. Ghajar. "Heat and mass transfer." A practical approach (2007).

[3] Wang, Pengfei, Jin Jiang, Shunyang Li, Xiangyu Luo, Shaojie Wang, and Wensheng Zhao. "An investigation of influence factor including different tube bundles on inclined elliptical fin-tube heat exchanger." International Journal of Heat and Mass Transfer 142 (2019): 118448. https://doi.org/10.1016/j.ijheatmasstransfer.2019.118448

[4] Tang, Linghong, Xueping Du, Jie Pan, and Bengt Sundén. "Air inlet angle influence on the air-side heat transfer and flow friction characteristics of a finned oval tube heat exchanger." International Journal of Heat and Mass Transfer 145 (2019): 118702. https://doi.org/10.1016/i.ijheatmasstransfer.2019.118702

[5] Naik, Hemant, and Shaligram Tiwari. "Thermal performance analysis of fin-tube heat exchanger with staggered tube arrangement in presence of rectangular winglet pairs." International Journal of Thermal Sciences 161 (2021): 106723. https://doi.org/10.1016/i.ijthermalsci.2020.106723

[6] Gupta, Arvind, Aditya Roy, Sachin Gupta, and Munish Gupta. "Numerical investigation towards implementation of punched winglet as vortex generator for performance improvement of a fin-and-tube heat exchanger." International Journal of Heat and Mass Transfer $149 \quad$ (2020): 119171. https://doi.org/10.1016/j.ijheatmasstransfer.2019.119171

[7] Modi, Ashish J., Navnath A. Kalel, and Manish K. Rathod. "Thermal performance augmentation of fin-and-tube heat exchanger using rectangular winglet vortex generators having circular punched holes." International Journal of Heat and Mass Transfer 158 (2020): 119724. https://doi.org/10.1016/i.ijheatmasstransfer.2020.119724

[8] Modi, Ashish J., and Manish K. Rathod. "Comparative study of heat transfer enhancement and pressure drop for fin-and-circular tube compact heat exchangers with sinusoidal wavy and elliptical curved rectangular winglet vortex generator." International Journal of Heat and Mass Transfer 141 (2019): $310-326$. https://doi.org/10.1016/i.ijheatmasstransfer.2019.06.088

[9] Kim, Nae-Hyun, and Soo-Hwan Kim. "Dry and wet air-side performance of a louver-finned heat exchanger having flat tubes." Journal of Mechanical Science and Technology 24, no. 7 (2010): 1553-1561. https://doi.org/10.1007/s12206-010-0409-1

[10] Zeeshan, Mohd, Sujit Nath, and Dipankar Bhanja. "Numerical study to predict optimal configuration of fin and tube compact heat exchanger with various tube shapes and spatial arrangements." Energy Conversion and Management 148 (2017): 737-752. https://doi.org/10.1016/j.enconman.2017.06.011

[11] Unger, Sebastian, Eckhard Krepper, Matthias Beyer, and Uwe Hampel. "Numerical optimization of a finned tube bundle heat exchanger arrangement for passive spent fuel pool cooling to ambient air." Nuclear Engineering and Design 361 (2020): 110549. https://doi.org/10.1016/j.nucengdes.2020.110549

[12] Toolthaisong, S., and N. Kasayapanand. "Effect of attack angles on air side thermal and pressure drop of the cross flow heat exchangers with staggered tube arrangement." Energy Procedia 34 (2013): 417-429. https://doi.org/10.1016/i.egypro.2013.06.770

[13] Gholami, A. A., Mazlan A. Wahid, and H. A. Mohammed. "Heat transfer enhancement and pressure drop for finand-tube compact heat exchangers with wavy rectangular winglet-type vortex generators." International Communications in Heat and Mass Transfer $54 \quad$ (2014): $132-140$. https://doi.org/10.1016/i.icheatmasstransfer.2014.02.016

[14] Sarangi, Shailesh Kumar, and Dipti Prasad Mishra. "Effect of tube shape on thermo-fluid performance of a winglet supported fin-and-tube heat exchanger having staggered tubes." Materials Today: Proceedings 41 (2021): $228-232$. https://doi.org/10.1016/i.matpr.2020.08.745

[15] Adam, A. Y., A. N. Oumer, Azri Alias, M. Ishak, and M. M. Noor. "Investigation of thermal-hydraulic performance in flat tube heat exchangers at various tube inclination angles." International Journal of Automotive \& Mechanical Engineering 14, no. 3 (2017). https://doi.org/10.15282/ijame.14.3.2017.12.0359

[16] Zainal, S., C. Tan, C. J. Sian, and T. J. Siang. "ANSYS simulation for Ag/HEG hybrid nanofluid in turbulent circular pipe." Journal of Advanced Research in Applied Mechanics 23, no. 1 (2016): 20-35.

[17] Välikangas, Turo, Shobhana Singh, Kim Sørensen, and Thomas Condra. "Fin-and-tube heat exchanger enhancement with a combined herringbone and vortex generator design." International Journal of Heat and Mass Transfer 118 (2018): 602-616. https://doi.org/10.1016/i.ijheatmasstransfer.2017.11.006 
[18] Phu, Nguyen Minh, and Pham Ba Thao. "Thermohydraulic Performance of a Fin and Inclined Flat Tube Heat Exchanger: A Numerical Analysis." CFD Letters 13, no. 7 (2021): 1-12. https://doi.org/10.37934/cfdl.13.7.112

[19] Roy, Rhitankar Saha, Lim Chong Lye, Lim Chin Guan, and Nor Mariah Adam. "ANSYS Simulation Study to Generate Pressure from Various Water-Wind Flow Conditions to Calculate Electricity Generated Using Piezoelectric Cells." CFD Letters 12, no. 9 (2020): 27-35. https://doi.org/10.37934/cfdl.12.9.2735

[20] Mohammed, Ahmed Yousif Adam. "Effect Of Tube Inclination Angle on The Thermal and Fluid Dynamic Performance of Flat Tube Heat Exchanger." PhD diss., Universiti Malaysia Pahang, 2017.

[21] Kalantari, Hosein, Seyed Ali Ghoreishi-Madiseh, Jundika C. Kurnia, and Agus P. Sasmito. "An analytical correlation for conjugate heat transfer in fin and tube heat exchangers." International Journal of Thermal Sciences 164 (2021): 106915. https://doi.org/10.1016/i.ijthermalsci.2021.106915

[22] Hoffmann-Vocke, J., J. R. Neale, and M. Walmsley. "The effect of fin-and-tube heat exchanger resistance on inlet flow maldistribution related pressure drop penalties." (2010). 